\title{
Priming of hand and foot response: is spatial attention to the body site enough?
}

\author{
Alison J. Wiggett ${ }^{1} \cdot$ Steven P. Tipper $^{2}$
}

Published online: 30 June 2015

(C) Psychonomic Society, Inc. 2015

\begin{abstract}
The purpose of the present study was to test whether we see evidence for body compatibility effects when viewing both familiar and unusual body postures. Specifically, in a task where colour targets have to be discriminated, we tested whether spatial orienting to a body site is sufficient for effects of body compatibility to emerge when viewing a task-irrelevant body or whether effects are dependent on whether or not we are able to adopt the viewed body posture. The results suggest that spatial orienting to a body site is insufficient; rather we argue that it is only postures that are familiar and we are easily able to adopt that can be processed fluently and influence target discrimination. This points to a key contribution of motor representations to body compatibility effects.
\end{abstract}

Keywords Body-part priming · Automatic imitation · Spatial priming $\cdot$ Motor expertise

\section{Introduction}

The observation of someone else's action facilitates similar actions in the observer: an intransitive action, such as a finger (Brass, Bekkering, Wohlschläger, \& Prinz, 2000) or hand (Press, Bird, Walsh, \& Heyes, 2008; Stürmer, Ascherleben, \& Prinz, 2000) movement will facilitate the response of the same finger/hand in the observer. Similar effects have been shown across body parts, such as hand/foot (Gillmeister,

Alison J. Wiggett

a.wiggett@bangor.ac.uk

1 School of Psychology, Adeilad Brigantia, Bangor University, Bangor, Wales, UK LL57 2AS

2 Department of Psychology, University of York, York, UK
Catmur, Liepelt, Brass, \& Heyes, 2008; Wiggett, Hudson, Tipper, \& Downing, 2011; Wiggett, Downing, \& Tipper, 2013) and hand/mouth (Leighton \& Heyes, 2010). This often is called automatic imitation as the effect of compatibility between observed and executed action is evident even when the observed action is irrelevant to the task (e.g., participants are responding to a colour or letter presented at the same time as the action). The underlying mechanism is thought to be the automatic activation of motor representations of topographically similar actions to those being observed - a process possibly mediated by the mirror neuron system (see Heyes, 2011 for recent review).

Bach, Peatfield, and Tipper (2007) reported two further important findings: first, "action" compatibility effects are evident even when static images of whole human bodies with no implied motion (images of a person standing up or sitting down) are viewed. Second, spatial attention has to be directed towards the body site for these body compatibility effects to emerge. Because the stimuli used by Bach et al. did not contain any actual to-be-imitated movement, we refer to the resulting effects as body compatibility effects rather than automatic imitation.

To investigate body-based compatibility effects, Bach et al. (2007) used naturalistic photographs of whole bodies. A coloured dot was superimposed on the hand or foot of the person in the photograph. This coloured dot instructed the participant to respond either with the hand or the foot (the location of the dot was task-irrelevant). The authors found that reaction times were faster on compatible trials (when the correct response was, e.g., a hand response and the dot was presented on the hand) compared with incompatible trials (e.g., a hand response when a colour dot was presented on the foot), suggesting that just orienting attention to a certain body part led to facilitation of the response with that same body part. However, there is a spatial confound in the Bach et al. study. 
The authors presented only typical body postures where the hands were spatially above the feet. This means that the body parts in the image had the same spatial relationship as the body parts the participant uses to respond (hands above feet). A number of studies looking at automatic imitation have explicitly tested for effects of spatial compatibility and whether automatic imitation effects can be explained simply by an alignment of body and spatial frames. These studies show that automatic imitation is not reducible to spatial compatibility (Brass, Bekkering \& Prinz, 2001; Catmur \& Heyes, 2011; Press et al., 2008; Wiggett et al., 2013). However, Bach et al.'s study did not control or test for this.

Using the same task as Bach et al. (2007), Welsh, McDougall, and Paulson (2014) recently went some way to discounting a purely spatial account. The authors were interested in the question of whether humans and animals are coded with respect to the same or different body systems. As in Bach et al., coloured targets were placed on either the hand or foot of a person, or on the front or hind limbs of different types of animals (cow, bear, monkey). Of most interest to the current paper was that the observed human body could either be in bipedal or quadrupedal position. Interestingly, the authors find evidence for body compatibility effects not only in the bipedal but also in some of the quadrupedal conditions. In other words, hand responses were faster to targets on the hand compared to targets on the foot, whereas the opposite was true for foot responses (faster to foot targets compared to hand targets) even when the viewed body was not in a typical, upright posture.

An important point in the Welsh et al. (2014) study was to demonstrate that the spatial location of the body-part played little role in the action priming. That is, in the quadrupedal position the hands and feet were on the same horizontal plane, which was orthogonal to the up-down relationship of the participant's hands-feet. Observing body-part specific priming in this condition supports the idea that the effect is not entirely dependent on spatial Simon-like cueing.

The current study investigates this further by examining the role of existing body representations in body compatibility effects. Note that the body postures used by Welsh et al. are ones that are highly familiar to people and easily adoptable. ${ }^{1}$ If body-part priming effects are related to body- and actionbased representations, then body postures that are not easily achievable will not activate simulation states in the viewer. We present images that match the "standard" body posture images in terms of body-parts primed (hands/feet) and the spatial relationship (hands above feet). Crucially, we also create body postures that participants cannot easily achieve (Fig. 1B). This

\footnotetext{
${ }^{1}$ Interestingly in Experiment 2 in Welsh et al (2014) participants were instructed to adopt this quadrupedal posture during the task. The authors found that the posture of the participant did not influence the pattern of compatibility effects (see also Fischer, 2005).
}

was done by using atypical body postures from the world of dance, yoga, and sport where the hands are spatially below the feet, and then inverting these images. This creates images that are similar to the typical body postures (Fig. 1A) in terms of the spatial arrangements of body parts. If the priming effects are caused by either, or both, the same body part attended and used for response, or a spatial overlap of hand above foot on both the screen and the participant's body, then significant priming effects must be observed. However, if it requires a motor representation that the participant must possess, condition B will not produce priming effects. Participants have limited motor representations of these poses as the poses were created by inverting images of atypical poses and as such are difficult to adopt. We would therefore argue that people can have little (motor) familiarity of these postures in this orientation and hence any activation of internal representations will not be achieved - or will only be achieved very slowly and therefore not affect the simple and relatively rapid task of colour discrimination.

Finally, in the experimental design to be described below we also included two other conditions. One was the unusual body postures adopted in situations, such as sport, dance, and yoga, which typical individuals cannot adopt without high levels of training. The other condition was typical body postures that were inverted. These two conditions were included to have a completely balanced design. Hence the conditions are described, and data shown in the figures, but they are not the central focus of our study. The key comparison is that of body postures that can be adopted versus those that are difficult to adopt. Crucially, the spatial arrangement of hands above feet is identical across these two conditions.

\section{Methods}

\section{Participants}

Twenty-nine Bangor University students (14 males, 15 females, mean age: 20.79 years) participated in exchange for course credits. Procedures were approved by the Ethics Committee of the School of Psychology at Bangor University.

\section{Materials}

The experiment was controlled by E-Prime run on a PC running Windows XP. The stimulus set was comprised of pictures of people in sporting or dancing poses. The images were taken from a variety of web-based sources. Twenty images showed a person in a "typical" body pose (i.e., hands above and feet below), and 20 images depicted an "atypical" body pose (i.e., hands below and feet above). Half the stimuli showed a male person and half a female person. Stimuli were presented upright and inverted. There were two main conditions of 
A. Familiar posture

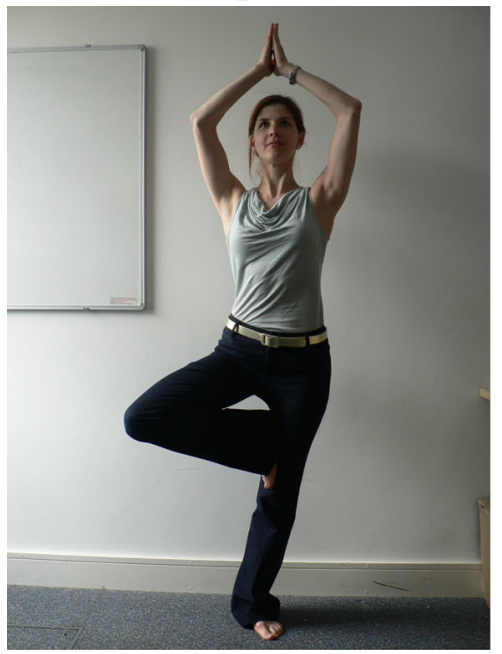

Fig. 1 Examples of familiar and unusual postures. The familiar posture (A) depicts a typical (hands above feet) body posture, where the image is presented upright. The unusual posture (B) is an atypical body posture where the image has been inverted. In terms of spatial relationship of

interest: 1) familiar body postures, which were images of typical body posture presented upright, and 2) unusual body postures, which were images of atypical body postures presented inverted. The remaining two (noncritical) conditions were typical images presented inverted and atypical images presented upright. Thus, the total stimulus set consisted of 80 images. The stimuli were presented in the centre of the screen and subtended approximately $10^{\circ}$ visual angle vertically and $10^{\circ}$ horizontally. A coloured target (red or blue) was superimposed on a hand or foot of the person in the image. Where the limb was off-centre, the target was placed to the left and the right of the midline with equal frequency. The target occupied approximately $1^{\circ}$ of visual angle.

Response times were measured by keyboard (space bar) for hand responses and with the use of a foot pedal (Savant Elite FS10J-USB, Kinesis) for foot responses. Participants used their right hand and right foot; the keyboard and foot pedal were positioned slightly to the right of the centre of the screen, allowing a natural, seated body position.

\section{Procedure}

Participants sat at a viewing distance of approximately $55 \mathrm{~cm}$ from the monitor. The participant's right hand was placed on the space bar of the keyboard, with their right foot on a foot pedal under the desk.

The trial sequence was based closely on Bach et al. (2007). Each trial started with a fixation-cross presented for $1400 \mathrm{~ms}$. The stimulus plus coloured target were then presented for up to $1100 \mathrm{~ms}$. If the participant made a correct response, the stimulus disappeared and was replaced by a blank screen for $1000 \mathrm{~ms}$ after which the next trial began. If the participant
B. Unusual posture

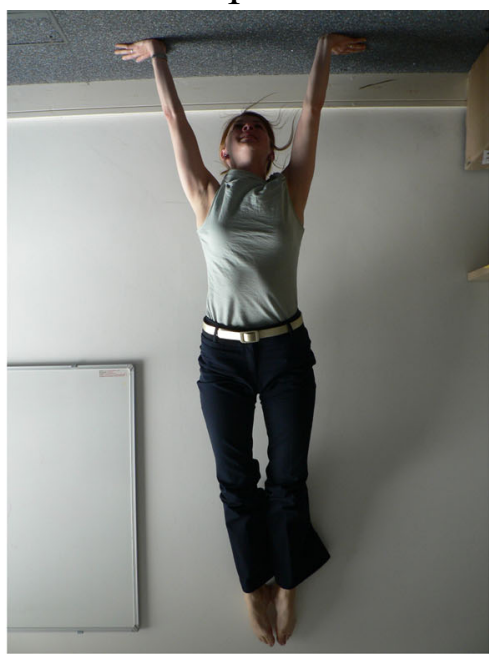

body parts (hands above feet) the two conditions are matched. Please note that, due to possible copyright infringements, these are not the actual stimuli used in the research

responded incorrectly, the word "incorrect" was presented for $1000 \mathrm{~ms}$. If the participant did not respond within the $1100 \mathrm{~ms}$ that the target stimulus was displayed, the message "too slow" appeared on the screen before the next trial started.

Participants were randomly assigned to one of two groups. Half the participants were given the instruction to respond with their hand to red targets and their foot to blue targets. The other half of participants were given the opposite instruction (note that for ease of communication "red" will henceforth refer to a hand response and blue to a foot response. Data were recorded for those participants who received the opposite instruction). Task instructions were presented on-screen and given verbally by the experimenter. The participant then completed a block of 10 practice trials. The main experiment consisted of 320 trials, split into 4 blocks of 80 trials. Each sporting/dance stimulus was presented once in each block. There were four different versions of each stimulus: a red target on the hand, a blue target on the hand, a red target on the foot, and a blue target on the foot. All typical and atypical stimuli were presented upright and inverted resulting in a total of 16 stimulus conditions (Fig. 2). This resulted in 20 trials per condition.

\section{Results}

Our main question concerns whether body compatibility effects are due to either a match of same body part attended and used for response or a spatial overlap of hand above foot on both the screen and the participant's body, or both. Alternatively, a match between body and spatial frames may not be necessary or sufficient for priming effects to be observed. Rather a participant's ability to adopt the body posture may be crucial for 
Effector-target compatible
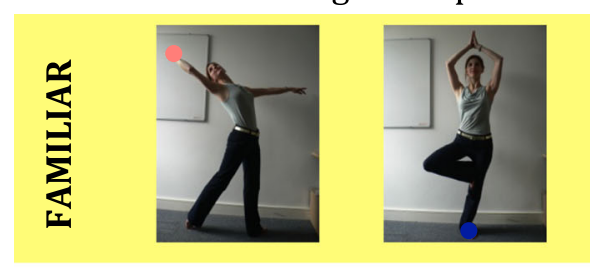

Effector-target incompatible
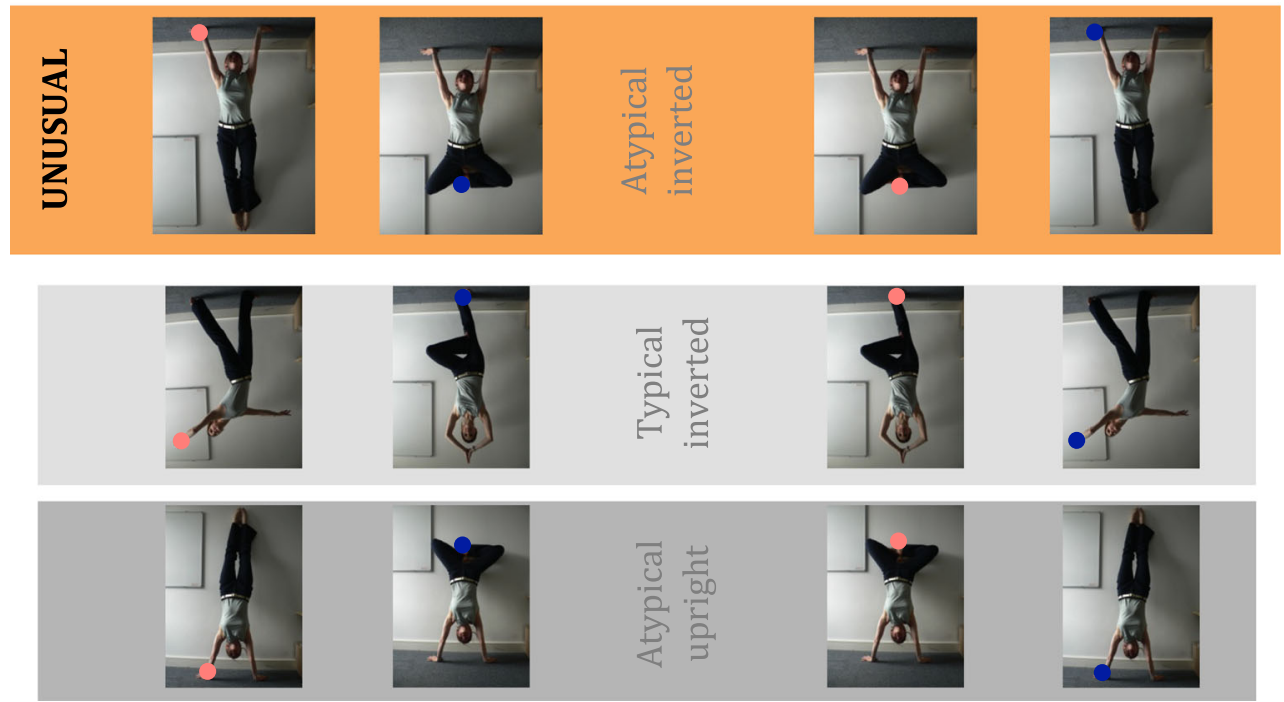

Fig. 2 Examples of postures presented in each of the sixteen conditions. The main conditions of interest were familiar (top row) and unusual (second row) postures. Red targets (columns 1 and 3) required a hand response; blue targets (columns 2 and 4 ) a foot response. Whether or not a given trial was effector-target compatible or incompatible therefore depended on the combination of target colour and the location of the target on the image. Please not that, due to possible copyright infringements, these are not the actual stimuli used in the research the emergence of these effects. To this end, we compared the conditions shown in Fig. 1 (familiar, unusual). Both these conditions possess the same body and spatial properties; they differ only in how familiar the postures are and therefore the ease with which they could be adopted.

One participant's data were excluded from all analyses due to the error rates being more than two standard deviations above the average number of errors across participants. For the analysis of reaction times (RTs), trials where the participant made the wrong response, responded too quickly $(<250 \mathrm{~ms})$, or did not respond within the time limit were eliminated $(6.5 \%)$. The results are shown in Fig. 3; the top panels show the results of our main conditions of interest (familiar and unusual postures). Our analysis focuses on these two conditions. However, the first step of the analysis was the overall ANOVA, including all factors. The data were entered into a repeated measures ANOVA with the factors familiarity (familiar/unusual), spatial configuration (upright, inverted,) response effector (hand/foot), and target location (hand/foot). Crucially, this revealed a significant four-way interaction $(F(1$, $27)=9.24, p<0.005, \eta^{2}=0.26$ ). This justifies the following focused analysis of our main conditions of interest.

The data for the two main conditions of interest were entered into a repeated measures ANOVA with the factors familiarity (familiar/unusual), response effector (hand/foot), and target location (hand/foot). Across familiar and unusual postures, there was a clear main effect for response effector $(F(1,27)=$ 152.06, $p<0.001, \eta^{2}=0.85$ ), reflecting the fact that hand responses $(M=532 \mathrm{~ms}, S D=13.2)$ were significantly faster than foot responses $(M=616 \mathrm{~ms}, S D=12.5)$. There was an interaction between response effector and target location $(F(1$, $27)=16.23, p<0.01, \eta^{2}=0.38$ ). Of most theoretical interest, there was a significant three-way interaction between the familiarity of the body posture, the response effector, and the target location $\left(F(1,27)=4.71, p<0.05, \eta^{2}=0.15\right)$. This reflects the fact that the body part priming effect was significant only for familiar but not unusual body postures. This is confirmed by post-hoc $t$ tests showing compatibility effects only for familiar postures: hand responses were faster to targets on the hand compared with on the foot $(t(27)=4.36, p<0.001)$, and foot responses were faster to targets on the foot compared with on the hand $(t(27)=2.65, p<0.05)$. There were no significant body part compatibility effects for unusual body postures.

The analysis of error rates (Table 1) across familiar and unusual body postures revealed a main effect of response effector $\left(F(1,27)=17.46, p<0.001, \eta^{2}=0.39\right)$ reflecting lower error rates for hand $(M=4.4, S D=0.66)$ compared with foot responses $(M=8.7, S D=1.04)$. There also was interaction between familiarity and target location $(F(1,27)=4.39, p<$ $\left.0.05, \eta^{2}=0.14\right)$. No other main effects or interactions were 


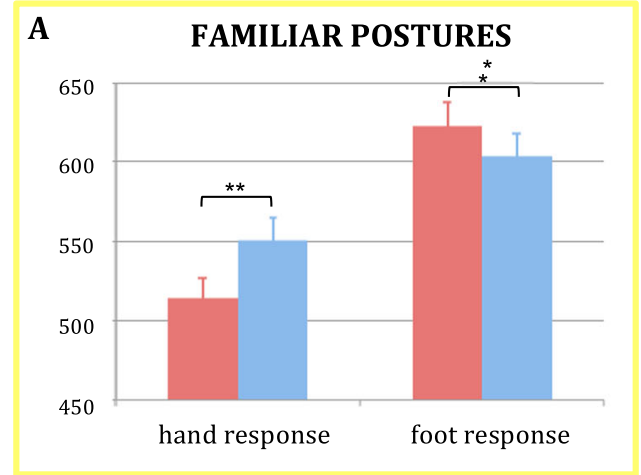

target on hand

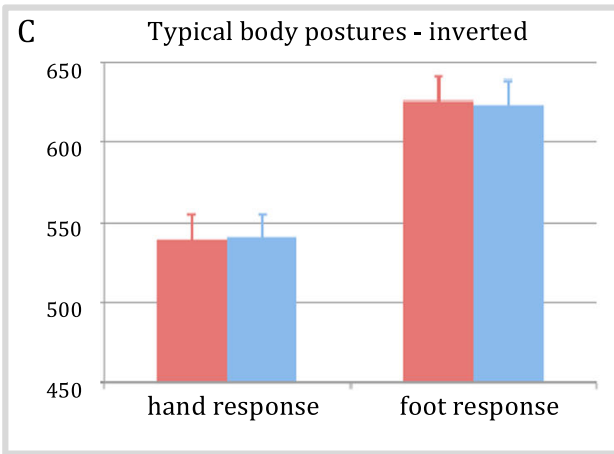

Fig. 3 Average mean reaction times for familiar (A) and unusual postures (B) (familiar postures = typical upright; unusual = atypical inverted). The remaining two conditions are shown in $\mathbf{C}$ (typical

significant. Overall, the error analysis suggests that body compatibility effects found for reaction times were not the result of a speed-accuracy trade-off where participants were responding more quickly, but with more errors, in one condition than the other.

Finally, for complete analysis, there were no significant compatibility effects for either of the other two conditions tested (typical inverted and atypical upright). Individual repeated measures ANOVAs for response effector by target location showed no significant interaction effects in reaction times $\left(\max F(1,27)=0.95, p>.05, \eta^{2}=0.03\right)$ or error rates $\left(\max F(1,27)=3.93, p>0.05, \eta^{2}=0.12\right)$. While these

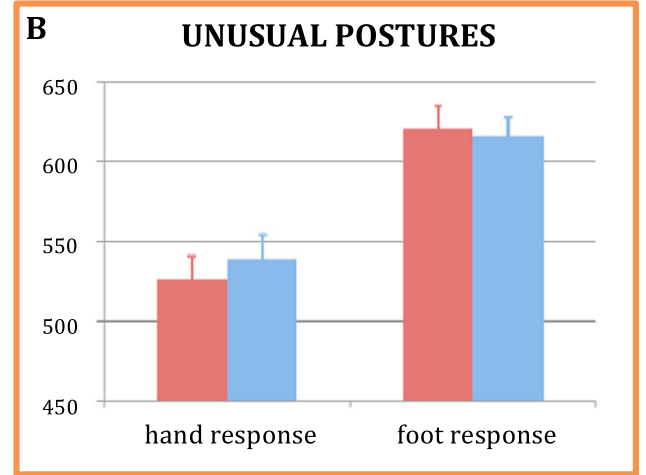

target on foot

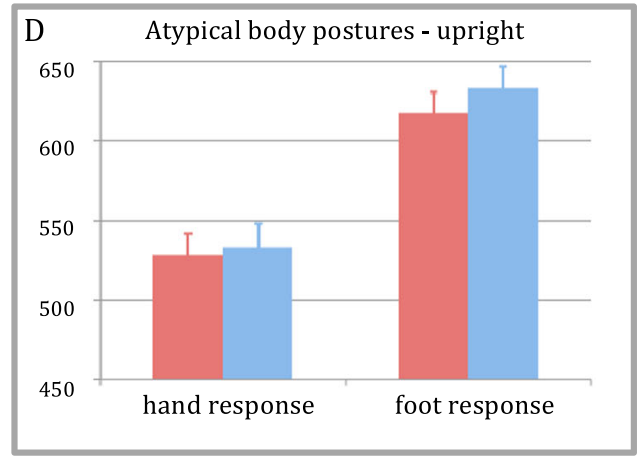

inverted) and D (atypical upright). Error bars represent \pm 1 standard error. $* * p<.001$;

$* p<0.05$

conditions were not central to our main question, the results clearly show that no body-part priming effects are evident when body and spatial frames are completely opposed. The results are in line with our conclusions that viewed body postures have to be familiar and adoptable for priming effects to emerge. However, the comparison of our two main conditions of interest is a stronger test of this due to the alignment of body and spatial frames.

To summarise, body-part priming effects are only detected when viewed bodies are in a "normal" orientation (i.e., hands above feet) and depict postures that people are able to easily adopt. A spatial overlap of hand above foot on both the screen

Table 1 Mean percentage of response errors (and standard errors)

\begin{tabular}{|c|c|c|c|c|}
\hline & \multicolumn{2}{|l|}{ Familiar postures } & \multicolumn{2}{|l|}{ Unusual postures } \\
\hline & Hand response & Foot response & Hand response & Foot response \\
\hline Target on hand & $2.85(0.83)$ & $8.21(1.39)$ & $4.64(1.12)$ & $9.12(1.15)$ \\
\hline \multirow[t]{3}{*}{ Target on foot } & $5.53(1.32)$ & $9.64(1.52)$ & $4.64(0.99)$ & $7.86(1.42)$ \\
\hline & Typical inverted & & Atypical upright & \\
\hline & Hand response & Foot response & Hand response & Foot response \\
\hline Target on hand & $3.39(0.72)$ & $10.18(1.73)$ & $3.04(0.74)$ & $8.93(1.73)$ \\
\hline Target on foot & $5.53(0.9)$ & $8.39(1.43)$ & $3.39(0.81)$ & $8.39(1.74)$ \\
\hline
\end{tabular}

Familiar and unusual postures appear in bold; top half of table. Typical inverted and atypical upright are shown in the bottom half of the table 
and participants body was not enough to produce priming. Thus, only familiar body postures presented upright lead to the emergence of body compatibility effects. This suggests that the viewer has to possess a motor representation of the viewed body posture.

\section{Discussion}

Previous work has shown that when viewing video clips of actions, they are imitated automatically even when the observed actions are irrelevant to the observer's task. Bach et al. (2007) showed that when viewing static images of actions, spatial attention has to be directed towards the acting body part for bodycompatibility effects to be evoked. A problem with a body-part explanation in the Bach et al. study is the possibility that spatial priming might play a part, as the visual images where hands were above feet were spatially compatible with the participants' body parts use to respond. However, the findings of Welsh et al. (2014) confirmed the Bach et al. results, but importantly by demonstrating body-part compatibility effects for observed quadrupedal postures Welsh et al. were able to discount spatial codes as the main, or at least only, cause of these effects.

The present study goes further by asking whether the ability to adopt a body posture is a critical factor in observing these priming effects. It specifically presents displays where a bodypart is primed and stimulus-response spatial codes match (hands above feet), but body posture can or cannot be adopted by participants. Our results show that a match between the response effector and the attended body site is in itself not enough to lead to body compatibility effects. Furthermore, a stimulus-response spatial overlap of hand above foot on both the screen and participant's body also is not enough to produce priming. Even though not relevant to the participant's task (which was simply to make a hand response to a red target and a foot response to a blue target, or vice versa), the observed body has to be in a conventional posture that participants are capable of adopting for the effects of body compatibility to emerge. The results suggest that in the short time (500$600 \mathrm{~ms}$ ) it takes the participant to respond to the coloured target it is not just the presence of a body, or even the orientation (hands above/feet below), that is processed but also something about the correspondence of the observed body posture to the action capability of the participant. Hence while observing bodies that are irrelevant to the main task of target colour discrimination, it is only well-established representations of bodies (i.e., familiar postures that participants can adopt) that are processed in a fast and fluent manner that influence performance.

Our results are in line with the literature pointing to a primary role of motor expertise for action perception. For example, the ability to perform a specialized set of motor skills results in increased perceptual accuracy when observing and discriminating the corresponding actions. This has been shown for basketball players predicting whether or not a shot was going to be on target (Aglioti, Cesari, Romani, \& Urgesi, 2008; see also Güldenpenning, Steinke, Koester, \& Schack, 2013 for a similar result in volleyball players), ballet dancers discriminating biological motion using point-light ballet moves (Calvo-Merino, Ehrenberg, Leung, \& Haggard, 2010), and for visual discrimination of newly learned gait patterns (Casile \& Giese, 2006). Also, neural activity in visuomotor areas tends to be stronger for movements that are in the observer's motor repertoire (Calvo-Merino, Glaser, Grèzes, Passingham, \& Haggard, 2005; see also CalvoMerino, Grèzes, Glaser, Passingham, \& Haggard, 2006; Cross, Hamilton, \& Grafton, 2006; Cross, Hamilton, Kraemer, Kelley, \& Grafton, 2009a; Cross, Kraemer, Hamilton, Kelley, \& Grafton, 2009b). Thus, the lack of body compatibility effect for unusual postures in the current study is likely due to the fact that the participants had little motor expertise of these postures and hence were less able to embody the unusual compared with the familiar body postures.

Acknowledgments The authors thank Geoffrey Taylor and Esther Alao for their help with data collection.

\section{References}

Aglioti, S. M., Cesari, P., Romani, M., \& Urgesi, C. (2008). Action anticipation and motor resonance in elite basketball players. Nature Neuroscience, 11, 1109-1116.

Bach, P., Peatfield, N. A., \& Tipper, S. P. (2007). Focusing on body sites: the role of spatial attention in action perception. Experimental Brain Research, 178, 509-517.

Brass, M., Bekkering, H., Wohlschläger, A., \& Prinz, W. (2000). Compatibility between observed and executed finger movements: comparing symbolic, spatial and imitative cues. Brain \& Cognition, 44, 124-143.

Brass, M., Bekkering, H., \& Prinz, W. (2001). Movement observation affects movement execution in a simple response task. Acta Psychologica, 106, 3-22.

Calvo-Merino, B., Glaser, D. E., Grèzes, J., Passingham, R. E., \& Haggard, P. (2005). Action observation and acquired motor skills: an FMRI study with expert dancers. Cerebral Cortex, 15, 12431249.

Calvo-Merino, B., Grèzes, J., Glaser, D. E., Passingham, R. E., \& Haggard, P. (2006). Seeing or doing? Influence of visual and motor familiarity in action observation. Current Biology, 16, 1905-1910.

Calvo-Merino, B., Ehrenberg, S., Leung, D., \& Haggard, P. (2010). Experts see it all: configural effects in action observation. Psychological Research, 74, 400-406.

Casile, A., \& Giese, M. A. (2006). Nonvisual motor training influences biological motion perception. Current Biology, 16, 69-74.

Catmur, C., \& Heyes, C. M. (2011). Time course analyses confirm the independence of imitative and spatial compatibility. Journal of Experimental Psychology: Human Perception and Performance, 37, 409-421.

Cross, E. S., Hamilton, A. F. C., \& Grafton, S. T. (2006). Building a motor simulation de novo: observation of dance by dancers. Neuroimage, 31, 1257-1267. 
Cross, E. S., Hamilton, A. F. C., Kraemer, D. J. M., Kelley, W. M., \& Grafton, S. T. (2009a). Dissociable substrates for body motion and physical experience in the human action observation network. European Journal of Neuroscience, 30, 1383-1392.

Cross, E. S., Kraemer, D. J. M., Hamilton, A. F. C., Kelley, W. M., \& Grafton, S. T. (2009b). Sensitivity of the action observation network to physical and observational learning. Cerebral Cortex, 19, 315326.

Fischer, M. H. (2005). Action simulation for others is not constrained by one's own postures. Neuropsychologia, 43, 28-34.

Gillmeister, H., Catmur, C., Liepelt, R., Brass, M., \& Heyes, C. (2008). Experience-based priming of body-parts: A study on action imitation. Brain Research, 1217, 157-170.

Güldenpenning, I., Steinke, A., Koester, D., \& Schack, T. (2013). Athletes and novices are differently capable to recognize feint and non-feint actions. Experimental Brain Research, 230, 333-343.

Heyes, C. M. (2011). Automatic imitation. Psychological Bulletin, 137, 463-483.
Leighton, J., \& Heyes, C. M. (2010). Hand to mouth: Automatic imitation across effector systems. Journal of Experimental Psychology: Human Perception and Performance, 36, 1174-1183.

Press, C., Bird, G., Walsh, E., \& Heyes, C. M. (2008). Automatic imitation of intransitive actions. Brain \& Cognition, 67, 44-50.

Stürmer, B., Aschersleben, G., \& Prinz, W. (2000). Correspondence effects with manual gestures and postures: A study of imitation. Journal of Experimental Psychology: Human Perception and Performance, 26, 1746-1759.

Welsh, T. N., McDougall, L., \& Paulson, S. (2014). The personification of animals: Coding of human and nonhuman body parts based on posture and function. Cognition, 132, 398-415.

Wiggett, A. J., Hudson, M., Tipper, S. P., \& Downing, P. E. (2011). Learning associations between action and perception: effects of incompatible training on body part and spatial priming. Brain \& Cognition, 76, 87-96.

Wiggett, A. J., Downing, P. E., \& Tipper, S. P. (2013). Facilitation and interference in spatial and body reference frames. Experimental Brain Research, 225, 116-131. 